\title{
Correspondence
}

\section{Aetiology of severe pneumonia in developing countries}

Sir,

Dr Ikeogu suggests, on the basis of his lung aspirate study of 40 children hospitalised in Bulawayo, that bacterial infection is not the commonest cause of severe pneumonia in children in Zimbabwe. ${ }^{1}$ This contrasts with published data from at least 13 lung aspirate studies from developing countries showing the importance of bacterial infections, in hospitalised children with severe (predominantly lobar) pneumonia. While geographical differences undoubtedly exist there are remarkable similarities in the results from these studies. The statement that 'negative bacterial growth from lung aspirates implies acute viral infection in most cases' should be read with caution. Bullowa's study of 211 bacteraemic pneumococcal pneumonias has suggested that lung aspiration has a false negative rate of at least $22 \%$. $^{2}$ Studies where viral cultures, immunofluorescence, or serology have been performed, together with lung aspiration or blood culture in children with severe pneumonia, have found evidence of viral infection only in approximately $30 \%$. If a genuinely low bacterial isolation rate in Bulawayo is to be established convincingly, serum or urine should be tested for antibacterial activity, as history of recent antibiotic treatment can often be unreliable. $^{2}$

Intervention trials from six countries have recently shown that improved case management with antibiotics may substantially reduce mortality from acute respiratory infections. ${ }^{3}$ This strategy is not necessarily one of increasing antibiotic prescription and hence escalating costs. Although viral and bacterial pneumonias cannot be differentiated reliably by clinical or radiological findings, simple clinical signs can be used to distinguish lower from upper respiratory infections thus allowing antibiotic delivery to be directed to the former group and potentially reducing antibiotic use in the community. ${ }^{4}$

We are concerned that the low mortality in this series suggests that these patients may not be typical of the majority of children in Zimbabwe and that the epidemic nature of respiratory infections requires that the minimum study period be one year (invasive pneumococcal disease in The Gambia appears to be noticeably seasonal). Furthermore, although Dr Ikeogu emphasises supportive care methods, it should be recognised that these are not yet proved to be effective in reducing mortality from pneumonia in developing countries. The weight of currently available evidence supports early antibiotic treatment as the primary intervention for all severe pneumonia in developing countries.

H CAMpbell and I M Forgie Medical Research Council Laboratories, PO Box 273, Fajara, The Gambia, West Africa
Dr Ikeogu comments:

Drs Campbell and Forgie raise a number of issues regarding my article on acute pneumonia. ${ }^{1}$ Space does not allow me to discuss all the points raised. They express surprise about the low mortality in the patients in the series. In our unit a lot of emphasis is placed on supportive care even in those with bacterial pneumonia. The supportive care principles are based on simple pathophysiological considerations but mechanical ventilation is used very infrequently in our unit and none of the 40 children was ventilated. The argument here is not distinguishing upper from lower respiratory infection but determining the aetiology of acute lower respiratory infection and therefore deciding when to use antibiotics. I consider the use of the suggested simple physical signs ${ }^{4}$ in arriving at this decision as an oversimplification, which is sometimes useful in situations where there are no doctors or paediatricians or facilities for appropriate investigations but which also, in my experience, often results in delayed referral to hospital often with disastrous consequences.

Also not treating upper respiratory infections because of absence of such simple signs as Drs Campbell and Forgie seem to suggest is dangerous in those areas with a high incidence of rheumatic fever. The difficulties in trying to simplify the management of respiratory infection in children are obvious and nothing can substitute experience backed up by appropriate investigations.

The purpose of my article was to indicate the possibility of regional differences in the patterns of pneumonia in developing countries and by so doing stimulate more investigations. Drs Campbell and Forgie agree with me that geographical differences and similarities do exist, a point reinforced by the absence of a single positive blood culture in any of the patients (this point was not mentioned in the article). The period of investigation included the peak period for respiratory infections and the population studied had no access to undocumented antibiotic exposure.

\section{References}

${ }^{1}$ Ikeogu MO. Acute pneumonia in Zimbabwe: bacterial isolates by lung aspiration. Arch Dis Child 1988;63:1266-7.

2 Shann F. Etiology of severe pneumonia in children in developing countries. Pediatr Infect Dis 1986;5:247-52.

${ }^{3}$ World Health Organisation. Case management of acute respiratory infections in children: intervention studies. Geneva: WHO, 1988. (WHO/ARI/88.2.)

${ }^{4}$ Cherian T, Jacob John T, Simoes E, Steinhoff MC, John M. Evaluation of simple clinical signs for the diagnosis of acute lower respiratory infections. Lancet 1988;ii:125-8.

\section{Seizures presenting as apnoea}

Sir,

Investigations of the infant with a seizure disorder presented by Navelet et al do not provide adequate data to state that the 